\title{
Hoffmannia uxpanapense (Rubiaceae), una nueva especie del sur de Veracruz y Oaxaca, México
}

\section{Hoffmannia uxpanapense (Rubiaceae), a new species from southern Veracruz and Oaxaca, Mexico}

\author{
Gonzalo Castillo-Campos² (D, Alma Patricia Bautista-Bello' (D)
}

\footnotetext{
1 Instituto de Ecología, A.C., Red de Biodiversidad y Sistemática, carretera Antigua a Coatepec Núm. 351, El Haya, 91070 Xalapa, Veracruz, México.

2 Autor para la correspondencia: gonzalo.castillo@inecol.mx
}

Citar como:

Castillo-Campos, G. y A. P. Bautista-Bello. 2017. Hoffmannia uxpanapense (Rubiaceae), una nueva especie del sur de Veracruz y Oaxaca, México. Acta Botanica Mexicana 119: 145-154. DOl: http://dx.doi. org/10.21829/abm119.2017.1236

Recibido: 11 de septiembre de 2015 .

Revisado: 28 de octubre de 2016

Aceptado: 26 de enero de 2017.

DOI:

http://dx.doi.org/10.21829/abm119.2017.1237

\section{ResUmeN:}

Antecedentes y Objetivos: La revisión de especímenes de herbario de Hoffmannia (Rubiaceae) y las intensivas exploraciones por los autores originaron el descubrimiento de nuevas especies de este género. Se describe e ilustra a Hoffmannia uxpanapense Cast.-Campos y Bautista-Bello como una nueva especie del sur de México, colectada en la selva alta perennifolia de Uxpanapa en Veracruz y Oaxaca.

Métodos: Se revisaron los ejemplares del género Hoffmannia para el estado de Veracruz, se elaboró una matriz de datos morfológicos y se llevó a cabo un análisis de conglomerados para diferenciar a las especies y detectar sus similitudes.

Resultados clave: Los ejemplares identificados como " $H$. wendtii" presentaron caracteres morfológicos diferentes a las especies ya descritas en este género. Dado que este nombre no ha sido publicado, se consideró como una especie nueva aún por describir formalmente. Este taxon muestra similitudes con $H$. cuneatissima, $H$. minuticarpa y $H$. arqueonervosa. Sin embargo, $H$. uxpanapense difiere por ser un arbusto trepador, con entrenudos del tallo más largos, inflorescencias más grandes y un mayor número de flores de color blanco por inflorescencia.

Conclusiones: Hoffmannia uxpanapense se integra a un grupo de especies endémicas de México (H. cuneatissima, H. minuticarpa y H. arqueonervosa), y se considera a este nuevo taxon como endémico de la zona sur de Veracruz y Oaxaca.

Palabras clave: arbusto trepador, especie endémica, Hoffmannia arqueonervosa, Hoffmannia cuneatissima, Hoffmannia minuticarpa.

\section{ABSTRACT}

Background and Aims: The revision of herbarium specimens of Hoffmannia (Rubiaceae) and the extensive exploration by the authors has resulted in the discovery of new species within this genus. Hoffmannia uxpanapense Cast.-Campos and Bautista-Bello is described and illustrated as a new species of the Rubiaceae family from southern Mexico, collected in the evergreen tropical forest of Uxpanapa in Veracruz and Oaxaca.

Methods: The specimens of the genus Hoffmannia of the state of Veracruz were studied, a matrix of morphological data was elaborated, and a cluster analysis was carried out to differentiate the species and to detect their similarities.

Key results: The specimens identified as " $H$. wendtii" presented different morphological characters to the species already described in this genus. As this name has not been published, it was considered as a new species that still needs to be formally described. This species shows similarities with $H$. cuneatissima, H. minuticarpa and $H$. arqueonervosa. Nevertheless, $H$. uxpanapense differs by being a climbing shrub, with larger internodes on the stem, larger inflorescences and a greater number of white flowers per inflorescence.

Conclusions: Hoffmannia uxpanapense belongs to a group of endemic species of Mexico (H. cuneatissima, H. minuticarpa and $H$. arqueonervosa), and this new taxon is considered as endemic of the southern zone of Veracruz and Oaxaca.

Key words: climbing shrub, endemic species, Hoffmannia arqueonervosa, Hoffmannia cuneatissima, Hoffmannia minuticarpa. 


\section{INTRODUCCIÓN}

Hoffmannia Sw. (Rubiaceae - Hamelieae) es uno de los géneros grandes de la familia y contiene entre 100 y 125 especies (Standley y Williams, 1975; Burger y Taylor, 2012), distribuidas principalmente en la región neotropical desde México y Las Antillas hasta Sudamérica (Standley, 1934; Standley y Williams, 1975; Dwyer, 1980; González y Poveda, 2004; Borhidi, 2006; Burger y Taylor, 2012). La mayoría de las especies se encuentran en las zonas montañosas del sur de México y en América Central (Williams, 1973; Taylor y Gereau, 2011).

Hoffmannia es un género poco conocido, dentro del mismo grupo es difícil de separar a los taxa, dado que en una misma población se puede presentar una variación sorprendente de varios de sus caracteres morfológicos, incluidos el tamaño, forma de las hojas y el color de las flores (Dwyer, 1969; Burger, 1999). Parte de esta variación se debe probablemente a los diferentes niveles de ploidía que se pueden presentar, particularmente a lo largo de un gradiente altitudinal (Kiehn, 1995).

El género Hoffmannia está representado en México por 34 especies de arbustos y hierbas (Borhidi, 2006; 2012), de las cuales cerca de $50 \%$ se encuen- tran en Veracruz. Considerando la variación morfológica de este género, los ejemplares de la nueva especie de Hoffmannia estuvieron ubicados bajo el nombre de $H$. wendtii, que tentativamente le había asignado D.H. Lorence. Este nombre no fue publicado porque Lorence después consideraba que este taxon y Hoffmannia rhizantha Standl. son una misma especie (com. pers.). Sin embargo, al hacer los análisis estadísticos con los caracteres morfológicos se pudo observar que $H$. rhizantha es diferente a las demás especies arbustivas localizadas en Veracruz (Fig. 1). De las 34 especies solo Hoffmannia minuticarpa Dwyer \& Lorence, H. cuneatissima B.L. Rob. y H. arqueonervosa Cast.-Campos presentan algunas características morfológicas similares a Hoffmannia sp. nov. (Figs. 1 y 2, Cuadro 1). Por lo tanto en este trabajo se describe un nuevo taxon del género Hoffmannia del sur de Veracruz y Oaxaca, México.

\section{MAteriales y MÉtOdos}

Se revisaron los especímenes del género Hoffmannia colectados para Veracruz y depositados en los herbarios más grandes de México (CHAPA, ENCB, MEXU y XAL), con un microscopio estereoscópico Carl Zeiss

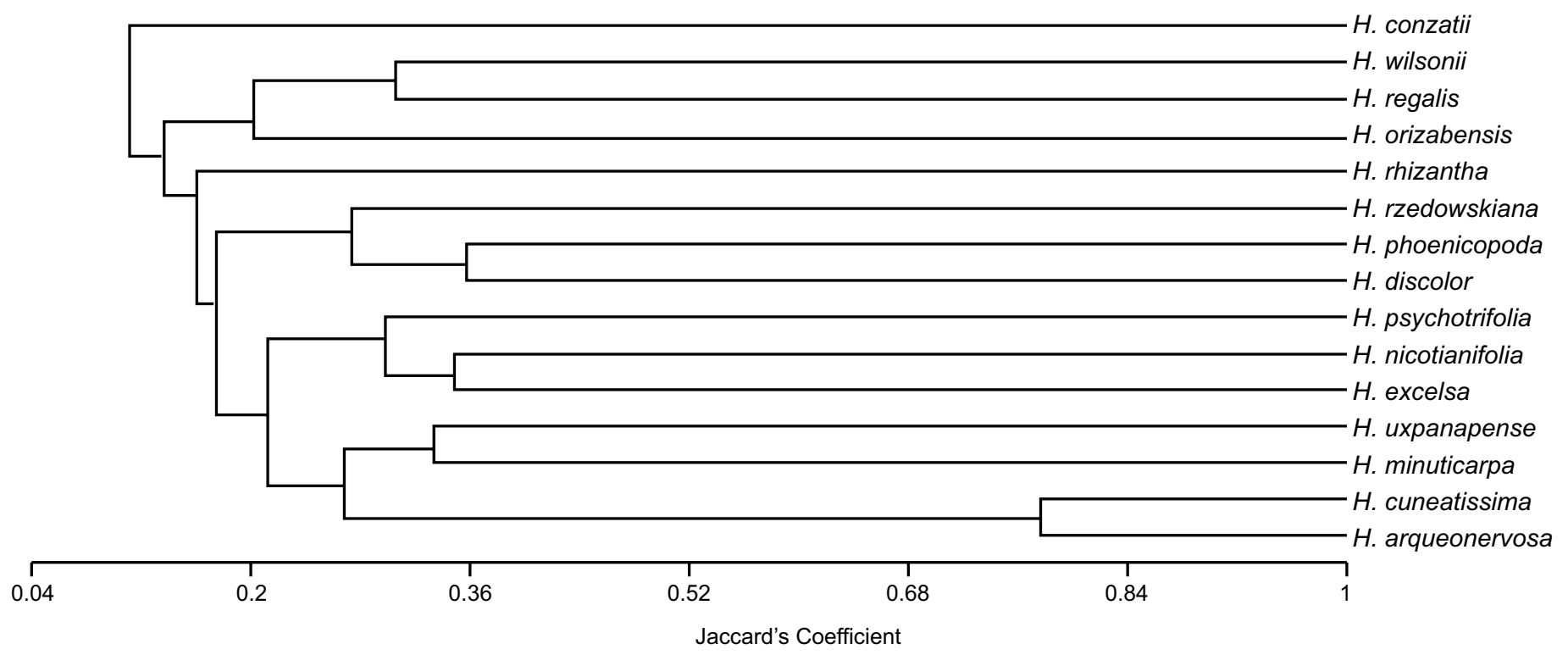

Figura 1: Dendrograma de agrupamiento de especies de acuerdo a la similitud de sus caracteres morfológicos. 
Cuadro 1: Comparación entre Hoffmannia uxpanapense Cast.-Campos y Bautista-Bello, H. arqueonervosa Cast.-Campos, H. cuneatissima B.L. Rob. y H. minuticarpa Dwyer \& Lorence.

\begin{tabular}{|c|c|c|c|c|}
\hline Caracteres & $\begin{array}{l}\text { H. uxpanapense Cast.- } \\
\text { Campos y Bautista- } \\
\text { Bello }\end{array}$ & $\begin{array}{l}\text { H. arqueonervosa Cast.- } \\
\text { Campos }\end{array}$ & H. cuneatissima B.L. Rob & $\begin{array}{l}\text { H. minuticarpa Dwyer \& } \\
\text { Lorence }\end{array}$ \\
\hline Altitud (m s.n.m.) & $100-200$ & 1600 & $1500-1600$ & $500-1200$ \\
\hline Tipo de vegetación & Selva alta perennifolia & Bosque mesófilo & Bosque mesófilo & $\begin{array}{l}\text { Selva alta perennifolia, } \\
\text { bosque mesófilo, } \\
\text { bosque de encino }\end{array}$ \\
\hline Forma biológica & Arbusto trepador & Arbusto & Arbusto & Arbusto \\
\hline Entrenudos (longitud, $\mathrm{cm}$ ) & $2-12$ & $1.5-8.5$ & $4-5$ & $1.2-4$ \\
\hline Haz de hojas & $\begin{array}{l}\text { Glabro, raramente con } \\
\text { pelos adpresos }\end{array}$ & $\begin{array}{l}\text { Glabro, ligeramente } \\
\text { lustroso }\end{array}$ & Ligeramente pubescente & Pubescente \\
\hline Envés & $\begin{array}{l}\text { Esparcidamente } \\
\text { punteado }\end{array}$ & Frecuentemente punteado & No punteado & No punteado \\
\hline Margen & Glabro & Glabro & Ciliado & Ligeramente ciliado \\
\hline Nervios laterales (pares) & $7-8(-10)$ & $9-12$ & $8-9$ & $10-18$ \\
\hline Peciolo (longitud cm) & $2.2-8$ & $2.5-9.8$ & $0.8-2$ & $1.5-5.5$ \\
\hline Inflorescencia (longitud cm) & $2.5-7$ & $2-2.8$ & 2 & $2.5-3$ \\
\hline Número de flores por inflorescencia & $6-15$ & $1-4(-5)$ & $4-5$ & $6-8$ \\
\hline $\begin{array}{l}\text { Pedúnculo de la inflorescencia } \\
\text { (longitud } \mathrm{mm} \text { ) }\end{array}$ & $20-50$ & $0.7-1.5$ & $10-11$ & $5.3-9.2$ \\
\hline Pedicelo (indumento) & Glabros & Glabros & Estrigosos & Estrigosos \\
\hline Corola (longitud mm) & $2.4-3.2$ & $7-11$ & $4-5$ & $5-6$ \\
\hline Corola (longitud del tubo mm) & 0.3 & $2-4$ & $1.4-2$ & $1.2-1.4$ \\
\hline Color de la corola & Blanca & $\begin{array}{l}\text { Amarilla, matizada de } \\
\text { rosa }\end{array}$ & Amarillenta & Amarillenta, verde \\
\hline Estigma & Bifurcado & Bifurcado & Linear & Linear \\
\hline
\end{tabular}

(Stemi 2000-C, Barrington, EUA). En la revisión del género Hoffmannia (Rubiaceae) para Veracruz, se encontraron cuatro especímenes con caracteres morfológicos diferentes a las especies ya descritas. Con 93 caracteres de las descripciones de 15 especies de Hoffmannia, registradas para Veracruz y los especímenes colectados en el sur de Veracruz y Oaxaca, se integró una matriz de presencia-ausencia (Cuadro 2) para evaluar su similitud mediante el índice de Jaccard (Sneath y Sokal, 1973). Utilizando el método de la media aritmética no ponderada (UPGMA), se generó un dendrograma de similitud con una escala de $0-1$, donde 0 representa $100 \%$ de di- ferencia en sus caracteres y 1, 100\% de similitud, con el apoyo del programa Multi Variate Statistical Package (MVSP), versión 3.1 (Kovach, 1999).

\section{Resultados}

El análisis de conglomerados agrupó a los especímenes que tenían el nombre de Hoffmannia wendtii, nom. herb., con las tres especies endémicas de México $(H$. cuneatissima) y Veracruz (H. arqueonervosa y H. minuticarpa), formando - con la nueva especie - un grupo de cuatro especies endémicas para México, con una similitud aproximada de 0.28 (Fig. 1). La especie más 


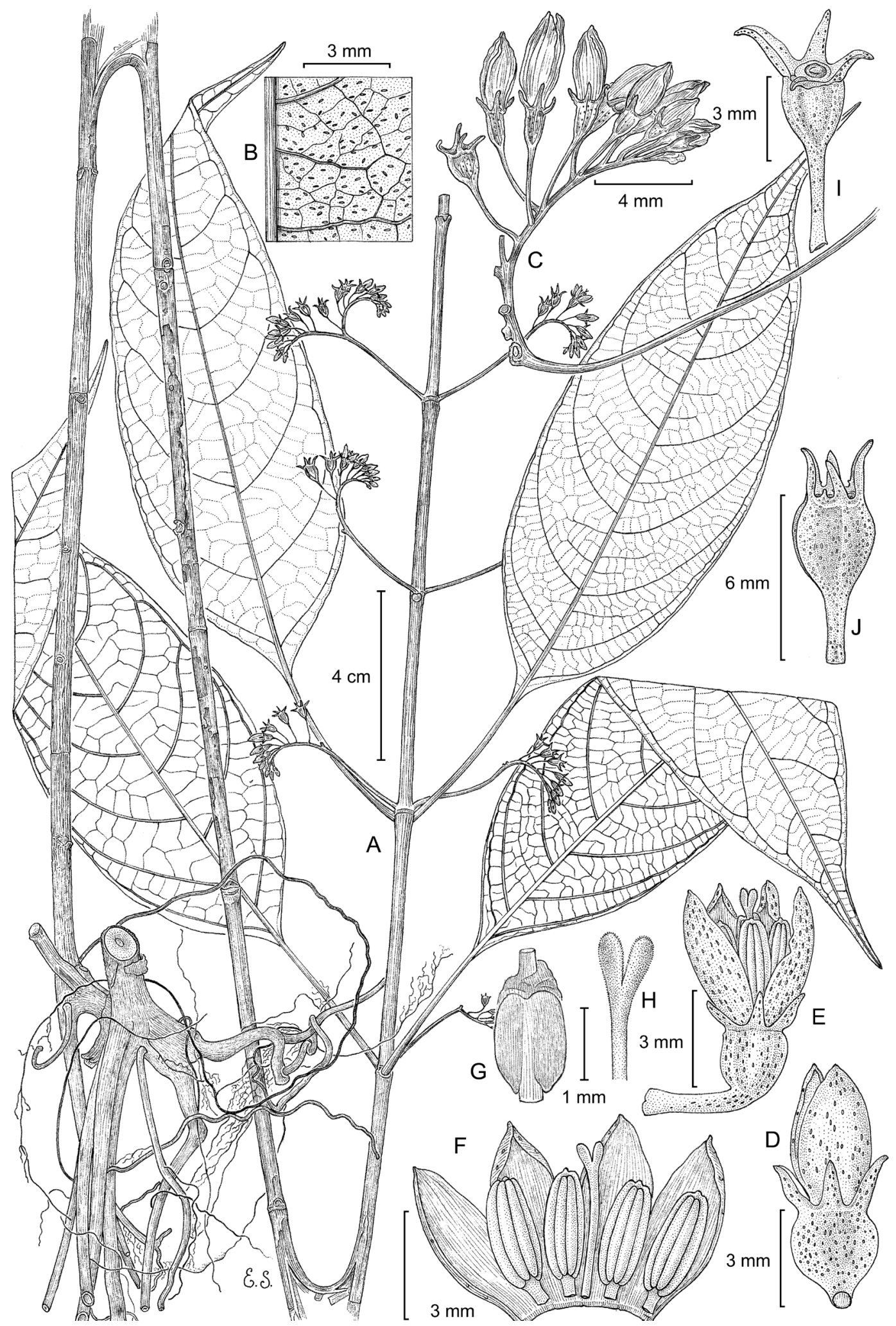

Figura 2: Hoffmannia uxpanapense Cast.-Campos y Bautista-Bello. A. rama mostrando la posición e inserción de las hojas, con flores y botones; B. envés de la hoja esparcidamente punteado; C. inflorescencia; D. botón; E. flor; F. botón abierto; G. ovario; H. estigma bifurcado; I, J. fruto inmaduro. Ilustración de Edmundo Saavedra, basada en los ejemplares T. Wendt 2588, 3405 (CHAPA, MEXU). 
Cuadro 2: Matriz de datos morfológicos de las especies del género Hoffmannia Sw. presentes en Veracruz, donde 0=ausencia, 1=presencia. En las columnas se consideró conveniente sustituir el nombre de las especies por un número progresivo: 1=H. arqueonervosa Cast.-Campos, $2=H$. conzattii B.L. Rob, 3=H. cuneatissima B.L. Rob, 4=H. discolor (Lem.) Hemsl., 5=H. excelsa (Kunth) K. Schum, 6=H. minuticarpa Dwyer \& Lorence, $7=H$. nicotianifolia (M. Martens \& Galeottii) L.O. Williams, 8=H. orizabensis Standl., 9=H. phoenicopoda K. Schum., 10=H. psychotrifolia (Benth.) Griseb., 11=H. rhizantha Stndl., 12=H. regalis (Hook.) Hemsl., 13=H. uxpanapense Cast.-Campos y Bautista-Bello, 14=H. wilsonii Standl., 15=H. rzedowskiana Cast.-Campos y Bautista-Bello \& Lorence.

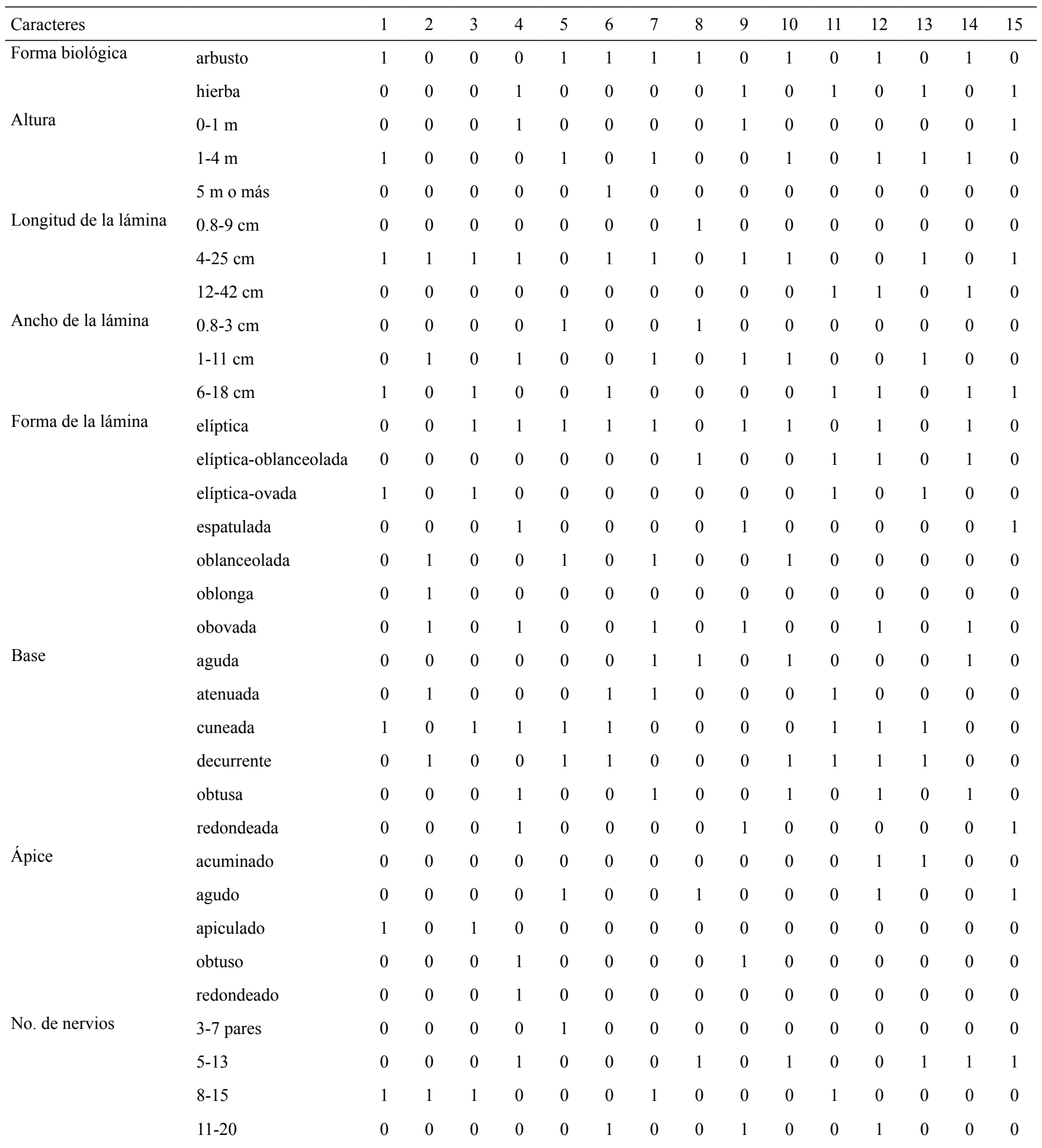


Cuadro 2: Continuación.

\begin{tabular}{|c|c|c|c|c|c|c|c|c|c|c|c|c|c|c|c|c|}
\hline Caracteres & & 1 & 2 & 3 & 4 & 5 & 6 & 7 & 8 & 9 & 10 & 11 & 12 & 13 & 14 & 15 \\
\hline \multirow[t]{2}{*}{ Tipo de nervadura } & broquidódroma & 0 & 0 & 0 & 0 & 0 & 0 & 0 & 0 & 0 & 0 & 1 & 1 & 0 & 0 & 0 \\
\hline & eucamptódroma & 1 & 0 & 1 & 1 & 1 & 0 & 1 & 0 & 1 & 1 & 0 & 0 & 1 & 0 & 1 \\
\hline \multirow[t]{5}{*}{ Peciolo } & sésil & 0 & 0 & 0 & 0 & 0 & 0 & 0 & 0 & 1 & 0 & 0 & 0 & 0 & 0 & 0 \\
\hline & $0.2-3 \mathrm{~cm}$ & 0 & 0 & 0 & 0 & 1 & 0 & 0 & 0 & 0 & 0 & 0 & 0 & 0 & 0 & 0 \\
\hline & $0.3-5 \mathrm{~cm}$ & 0 & 0 & 0 & 0 & 0 & 1 & 0 & 0 & 0 & 1 & 0 & 0 & 0 & 0 & 0 \\
\hline & $0.4-5.5 \mathrm{~cm}$ & 0 & 0 & 0 & 1 & 0 & 0 & 1 & 1 & 0 & 0 & 0 & 0 & 0 & 0 & 1 \\
\hline & $1.2-12 \mathrm{~cm}$ & 1 & 1 & 1 & 0 & 0 & 0 & 0 & 0 & 0 & 0 & 1 & 1 & 1 & 1 & 0 \\
\hline \multirow[t]{3}{*}{ Estípulas } & $1-2 \mathrm{~mm}$ & 1 & 0 & 1 & 0 & 1 & 1 & 1 & 0 & 0 & 1 & 0 & 0 & 1 & 0 & 1 \\
\hline & $2-4 \mathrm{~mm}$ & 0 & 0 & 0 & 1 & 0 & 0 & 0 & 0 & 0 & 0 & 0 & 1 & 0 & 0 & 0 \\
\hline & $3-10 \mathrm{~mm}$ & 0 & 0 & 0 & 0 & 0 & 0 & 0 & 0 & 1 & 0 & 1 & 0 & 0 & 0 & 0 \\
\hline \multirow[t]{2}{*}{ Forma de las estípulas } & elíptica & 0 & 0 & 0 & 0 & 0 & 0 & 0 & 0 & 1 & 0 & 0 & 0 & 0 & 0 & 0 \\
\hline & triangular & 1 & 0 & 1 & 1 & 1 & 1 & 0 & 0 & 0 & 1 & 1 & 1 & 1 & 0 & 1 \\
\hline \multirow{3}{*}{$\begin{array}{l}\text { Tamaño de la } \\
\text { inflorescencia }\end{array}$} & $1-3 \mathrm{~cm}$ & 1 & 0 & 1 & 0 & 1 & 1 & 1 & 0 & 0 & 1 & 0 & 0 & 1 & 0 & 0 \\
\hline & $2-7 \mathrm{~cm}$ & 0 & 0 & 0 & 0 & 0 & 0 & 0 & 0 & 1 & 0 & 0 & 1 & 0 & 0 & 0 \\
\hline & $3-15 \mathrm{~cm}$ & 0 & 0 & 0 & 1 & 0 & 0 & 0 & 0 & 0 & 0 & 1 & 0 & 0 & 0 & 0 \\
\hline \multirow[t]{2}{*}{ Tipo de inflorescencia } & cimosa & 0 & 0 & 0 & 1 & 0 & 0 & 1 & 0 & 1 & 0 & 0 & 1 & 1 & 1 & 0 \\
\hline & fasciculada & 0 & 0 & 0 & 0 & 0 & 0 & 0 & 0 & 0 & 1 & 0 & 0 & 0 & 1 & 0 \\
\hline \multirow[t]{5}{*}{ Pedúnculo } & $0.2-0.8 \mathrm{~cm}$ & 0 & 0 & 0 & 0 & 0 & 0 & 0 & 0 & 0 & 1 & 0 & 0 & 0 & 0 & 0 \\
\hline & $0.5-2 \mathrm{~cm}$ & 0 & 0 & 0 & 0 & 1 & 0 & 1 & 1 & 0 & 0 & 0 & 0 & 0 & 0 & 0 \\
\hline & $0.5-2.8 \mathrm{~cm}$ & 1 & 0 & 1 & 0 & 0 & 0 & 0 & 0 & 0 & 0 & 0 & 1 & 0 & 0 & 0 \\
\hline & $2-11 \mathrm{~cm}$ & 0 & 0 & 0 & 1 & 0 & 1 & 0 & 0 & 1 & 0 & 1 & 0 & 1 & 0 & 1 \\
\hline & $2-30 \mathrm{~cm}$ & 0 & 0 & 0 & 0 & 0 & 0 & 0 & 0 & 0 & 1 & 0 & 0 & 0 & 0 & 0 \\
\hline \multirow[t]{2}{*}{ Pedicelo } & $1-7 \mathrm{~mm}$ & 0 & 0 & 1 & 1 & 0 & 1 & 0 & 1 & 1 & 0 & 0 & 1 & 1 & 0 & 0 \\
\hline & $3-14 \mathrm{~mm}$ & 0 & 0 & 0 & 0 & 1 & 0 & 1 & 0 & 0 & 0 & 1 & 0 & 0 & 0 & 0 \\
\hline \multirow[t]{4}{*}{ Cáliz } & $0.2-0.8 \mathrm{~mm}$ & 0 & 0 & 0 & 0 & 0 & 1 & 0 & 0 & 0 & 0 & 0 & 1 & 0 & 0 & 0 \\
\hline & $0.3-1.5 \mathrm{~mm}$ & 1 & 0 & 1 & 0 & 0 & 0 & 1 & 0 & 0 & 0 & 1 & 0 & 0 & 1 & 0 \\
\hline & $0.5-1(3) \mathrm{mm}$ & 0 & 0 & 0 & 0 & 1 & 0 & 0 & 0 & 0 & 1 & 0 & 0 & 0 & 0 & 0 \\
\hline & $1-3(6) \mathrm{mm}$ & 0 & 0 & 0 & 1 & 0 & 0 & 0 & 0 & 1 & 0 & 0 & 0 & 1 & 0 & 0 \\
\hline \multirow[t]{4}{*}{ Forma } & agudo & 0 & 0 & 0 & 0 & 0 & 0 & 0 & 1 & 0 & 0 & 0 & 0 & 0 & 1 & 0 \\
\hline & deltado & 0 & 0 & 0 & 0 & 0 & 0 & 1 & 0 & 0 & 0 & 0 & 0 & 0 & 0 & 0 \\
\hline & linear & 0 & 0 & 0 & 0 & 1 & 0 & 0 & 0 & 0 & 0 & 0 & 0 & 0 & 0 & 0 \\
\hline & oblongo & 0 & 0 & 0 & 0 & 0 & 0 & 0 & 0 & 1 & 0 & 0 & 0 & 0 & 0 & 0 \\
\hline \multirow[t]{4}{*}{ Color de la flor } & amarilla & 1 & 0 & 1 & 0 & 1 & 1 & 1 & 0 & 1 & 1 & 0 & 1 & 0 & 0 & 0 \\
\hline & anaranjada & 0 & 0 & 0 & 0 & 0 & 0 & 0 & 0 & 0 & 1 & 0 & 0 & 0 & 0 & 0 \\
\hline & blanca & 0 & 0 & 0 & 0 & 1 & 0 & 0 & 0 & 0 & 1 & 0 & 0 & 0 & 0 & 0 \\
\hline & matizado de rosado & 1 & 0 & 1 & 0 & 0 & 0 & 0 & 0 & 0 & 0 & 0 & 0 & 0 & 0 & 0 \\
\hline
\end{tabular}


Cuadro 2: Continuación.

\begin{tabular}{|c|c|c|c|c|c|c|c|c|c|c|c|c|c|c|c|c|}
\hline Caracteres & & 1 & 2 & 3 & 4 & 5 & 6 & 7 & 8 & 9 & 10 & 11 & 12 & 13 & 14 & 15 \\
\hline & matizado de verde & 0 & 0 & 0 & 0 & 0 & 1 & 1 & 0 & 0 & 0 & 0 & 0 & 0 & 0 & 0 \\
\hline & roja & 0 & 0 & 0 & 1 & 1 & 0 & 1 & 0 & 1 & 0 & 1 & 0 & 0 & 0 & 0 \\
\hline & rosada & 0 & 0 & 0 & 1 & 1 & 0 & 1 & 0 & 0 & 0 & 0 & 1 & 0 & 0 & 0 \\
\hline & verde & 0 & 0 & 0 & 0 & 0 & 0 & 0 & 0 & 0 & 0 & 0 & 1 & 0 & 0 & 0 \\
\hline \multirow[t]{4}{*}{ Tubo } & $0.5-3 \mathrm{~mm}$ & 0 & 0 & 0 & 0 & 1 & 1 & 1 & 0 & 1 & 0 & 1 & 0 & 1 & 0 & 0 \\
\hline & $2-4 \mathrm{~mm}$ & 1 & 1 & 1 & 1 & 0 & 0 & 0 & 1 & 0 & 0 & 0 & 1 & 0 & 1 & 0 \\
\hline & $4-9 \mathrm{~mm}$ & 0 & 0 & 0 & 0 & 0 & 0 & 0 & 0 & 0 & 1 & 0 & 0 & 0 & 0 & 0 \\
\hline & $8-9 \mathrm{~mm}$ & 0 & 0 & 0 & 0 & 0 & 0 & 0 & 0 & 1 & 0 & 0 & 0 & 0 & 0 & 0 \\
\hline \multirow[t]{5}{*}{ Lobos } & $0.2-1 \mathrm{~mm}$ & 0 & 0 & 0 & 0 & 0 & 1 & 0 & 0 & 0 & 0 & 0 & 0 & 0 & 0 & 0 \\
\hline & $1.5-3.5 \mathrm{~mm}$ & 0 & 0 & 0 & 1 & 1 & 0 & 0 & 0 & 0 & 1 & 0 & 1 & 1 & 0 & 0 \\
\hline & $2-4 \mathrm{~mm}$ & 0 & 0 & 0 & 0 & 0 & 1 & 0 & 0 & 0 & 0 & 0 & 0 & 1 & 0 & 1 \\
\hline & $3-5 \mathrm{~mm}$ & 1 & 0 & 1 & 0 & 0 & 0 & 1 & 0 & 1 & 0 & 0 & 0 & 0 & 0 & 0 \\
\hline & $4-8 \mathrm{~mm}$ & 0 & 0 & 0 & 0 & 1 & 0 & 1 & 0 & 1 & 0 & 0 & 1 & 0 & 0 & 0 \\
\hline Anteras & $6-12 \mathrm{~mm}$ & 0 & 0 & 0 & 1 & 0 & 0 & 0 & 0 & 0 & 1 & 1 & 0 & 0 & 0 & 0 \\
\hline \multirow[t]{5}{*}{ Forma del fruto } & elipsoidales & 0 & 0 & 0 & 0 & 0 & 0 & 0 & 0 & 1 & 1 & 1 & 0 & 0 & 0 & 0 \\
\hline & globosos & 0 & 0 & 0 & 0 & 1 & 1 & 0 & 0 & 0 & 0 & 0 & 0 & 1 & 0 & 0 \\
\hline & lanceolados & 0 & 0 & 0 & 0 & 0 & 0 & 0 & 0 & 0 & 1 & 0 & 0 & 0 & 0 & 0 \\
\hline & obovados & 0 & 0 & 0 & 0 & 1 & 0 & 0 & 0 & 0 & 0 & 0 & 0 & 0 & 0 & 0 \\
\hline & subgloboso & 0 & 0 & 0 & 1 & 0 & 0 & 1 & 0 & 0 & 1 & 0 & 1 & 0 & 0 & 1 \\
\hline \multirow[t]{5}{*}{ Color del fruto } & fuchsia & 0 & 0 & 0 & 0 & 0 & 0 & 0 & 0 & 0 & 1 & 0 & 0 & 0 & 0 & 0 \\
\hline & lila-morado & 0 & 0 & 0 & 0 & 0 & 0 & 0 & 0 & 0 & 0 & 0 & 1 & 0 & 0 & 0 \\
\hline & negro & 0 & 0 & 1 & 0 & 0 & 0 & 0 & 0 & 0 & 0 & 0 & 0 & 0 & 0 & 0 \\
\hline & rojo & 0 & 0 & 0 & 0 & 1 & 0 & 1 & 0 & 1 & 0 & 0 & 1 & 0 & 0 & 1 \\
\hline & rosado & 0 & 0 & 0 & 0 & 0 & 0 & 1 & 0 & 0 & 1 & 0 & 0 & 0 & 0 & 0 \\
\hline
\end{tabular}

relacionada con Hoffmannia sp. nov. es H. minuticarpa con una similitud aproximada de 0.33 (Fig. 1). Considerando la similitud que presenta Hoffmannia sp. nov. con las especies localizadas en Veracruz, se puede definir claramente como un nuevo taxon (Fig. 1, Cuadro 1). Todas las especies de Hoffmannia que ocurren en Veracruz (H. arqueonervosa, $H$. conzattii B.L. Robins., H. cuneatissima, H. excelsa (Kunth) K. Schum., H. minuticarpa, H. nicotianifolia (Mart. \& Gal.) L.O. Williams, H. orizabensis Standl., H. psychotrifolia
(Benth. in Oerst.) Griseb., H. regalis (Hook. f.) Hemsl., $H$. rhizantha, $H$. wilsonii Standl. y Hoffmannia sp. nov.) son arbustos, con excepción de H. rzedowskiana Cast.-Campos, Bautista-Bello y Lorence, H. phoenicopoda K. Schum. y H. discolor (Lem.) Hemsl. que son hierbas. Todos estos taxa solo crecen en vegetación primaria, por lo que están fuertemente amenazados en el estado de Veracruz, si las comunidades vegetales donde se encuentran localizados desaparecen por el cambio de uso del suelo. 
Hoffmannia uxpanapense Cast.-Campos y Bautista-Bello, sp. nov. Fig. 2.

TIPO: MÉXICO. Veracruz, municipio de Uxpanapa, $8.2 \mathrm{~km}$ al norte de la terracería La Laguna - Río Grande, sobre el camino a ejido Belisario Domínguez, selva alta perennifolia, $130 \mathrm{~m}$ s.n.m., 16.VII.1980, T. Wendt et al. 2588 (holotipo: CHAPA!, isotipo: MEXU!).

Hoffmannia uxpanapense is related to $H$. minuticarpa; however, it differs by being a climbing shrub, with stem internodes 2-12 cm long, petioles 2.2-8 cm long, ovate-elliptical blades, 7-8(-10) pairs of lateral veins, inflorescences $2.5-7 \mathrm{~cm}$ long, with 6-15 flowers per inflorescence, inflorescence peduncles $2-5 \mathrm{~cm}$ long, glabrous floral pedicels, white flowers, corolla $2.4-3.2 \mathrm{~mm}$ long and the bifurcate stigma.

Planta arbustiva, trepadora, 1-3 m de alto, tallo erecto, glabro, entrenudos 2-12 cm de longitud; hojas opuestas, ascendentes, láminas ovada-elípticas, 8-17(21) $\times 3.5-7.5 \mathrm{~cm}$, haz glabro, en ocasiones con pelos adpresos, lustroso, envés esparcidamente punteado, verde pálido, margen entero, ápice acuminado, base cuneada a decurrente, nerviación eucamptódroma, nervios laterales 7-8(-10) pares, peciolo $2.2-8 \mathrm{~cm}$ de longitud, $1 \mathrm{~mm}$ de grueso, glabro, punteado, ligeramente acostillado, estípulas triangulares, $1.1 \times 1.1 \mathrm{~mm}$ de ancho en la base; inflorescencia axilar, cimosa, 2.5-7 $\times 2.5-3.5 \mathrm{~cm}, 1$ inflorescencia por nudo; flores blancas, 6-15 por inflorescencia, pedúnculos de $2-5 \mathrm{~cm}$ de longitud, $1 \mathrm{~mm}$ de grueso, pedicelos de $2-5 \times 2 \mathrm{~mm}$, glabros; cáliz 4-lobado, lóbulos triangulares, 1.1-2 $\times$ 0.2-0.4 mm; tubo de la corola $0.3 \times 0.4 \mathrm{~mm}$; corola de 2.4-3.2 mm de longitud, glabra externa e internamente, lóbulos 4, de 3.4-4.1 × $1.2 \mathrm{~mm}$; estambres 4, 3-3.2 mm de longitud, anteras $2.3 \times 0.4 \mathrm{~mm}$, glabras, basifijas, filamento $0.3 \mathrm{~mm}$ de longitud, estilo $3.3 \mathrm{~mm}$ de longitud, estigma bifurcado, de $0.4 \times 1.1 \mathrm{~mm}$, lóbulos $0.3 \mathrm{~mm}$ de longitud; ovario 1.1-2.3 × 1.1-2 mm; fruto abayado, verde, 2.4-4 $\mathrm{mm}$ de longitud, glabro.
Especímenes adicionales examinados: MÉXICO. Veracruz, municipio de Uxpanapa, lomas al S del Poblado Once, cerca de $27 \mathrm{~km}$ al E de La Laguna, T. Wendt et al. 3405 (CHAPA, MEXU), 4031 (MEXU). Oaxaca, municipio de Santa María Chimalapa, San Antonio Nuevo Paraíso, a $1 \mathrm{~km}$ en línea recta al W, por el camino al Plan de la Ceiba, J. E. Rivera H. et al. 1397 (MEXU), 1402 (MEXU).

Fenología: Hoffmannia uxpanapense florece de junio a agosto y fructifica de septiembre a noviembre.

Eponimia: El nombre de la nueva especie está dedicado a la localidad de Uxpanapa, Veracruz y límites con Los Chimalapas, Oaxaca, lugar donde crece el taxon.

Distribución y hábitat: Después de varias salidas de campo para colectar más especímenes en los límites de Veracruz con Oaxaca y no encontrar la planta, se llegó a la conclusión de que Hoffmannia uxpanapense restringe su distribución a la selva alta perennifolia del sur de Veracruz, probablemente endémica de esa región de México.

Hoffmannia uxpanapense es una especie que forma parte del estrato arbustivo de la selva alta perennifolia en los afloramientos calizos de la zona de Uxpanapa, Veracruz y en los Chimalapas, Oaxaca, tanto en áreas de vegetación secundaria como en las bien conservadas de la selva alta perennifolia sensu Miranda y Hernández X. (1963), en altitudes de 100 a $200 \mathrm{~m}$. Las zonas tienen generalmente un clima tropical lluvioso, con temperatura media anual del mes más frío mayor a $18{ }^{\circ} \mathrm{C}$ (García, 1988). En la selva alta se asocia en el estrato arbóreo principalmente con Cedrela odorata L., Dialium guianense (Aubl.) Sandwith, Guarea glabra Vahl, Guarea tonduzii C. DC., Guatteria spp., Lonchocarpus spp., Poulsenia armata (Miq.) Standl., Pouteria spp., Pterocarpus spp., Quararibea funebris (La Llave) Vischer y Rinorea guatemalensis (S. Watson) Bartlett. En el estrato arbustivo los taxa más comunes son Ardisia pellucida Oerst., Leandra dichotoma 
(Pav. ex D. Don) Cogn., Neea psychotrioides Donn. Sm., Psychotria elata (Sw.) Hammel y Psychotria deflexa DC. El estrato herbáceo está caracterizado por Anthurium pedatoradiatum Schott, Aphelandra aurantiaca (Scheidw.) Lindl., Begonia pustulata Liebm., Blechnum schiedeanum (Schltdl. ex C. Presl) Hieron., Chamaedorea elatior Mart., Hoffmannia discolor (Lem.) Hemsl. y Reinhardtia gracilis (H. Wendl.) Drude ex Dammer.

\section{DISCUSIÓN Y CONCLUSIÓN}

Hoffmannia uxpanapense es un arbusto endémico del sur de Veracruz y Oaxaca, confinado a altitudes bajas en las reducidas selvas tropicales de los límites de Veracruz con Los Chimalapas, Oaxaca. El ser un arbusto trepador lo hace distintivo de las demás especies arbustivas conocidas para México y Veracruz. Está relacionada con $H$. minuticarpa, que también es un arbusto endémico de las selvas tropicales, bosque mesófilo de montaña y encinares de la sierra de Los Tuxtlas, Veracruz, que habita en altitudes de 500 a $1200 \mathrm{~m}$. Sin embargo, las diferencias en sus caracteres morfológicos de Hoffmannia uxpanapense con H. minuticarpa son de hasta $70 \%$ de acuerdo al análisis estadístico y con las demás especies arbustivas localizadas en el estado de $25 \%$ aproximadamente (Fig. 1), incluyendo a las otras tres especies endémicas (Hoffmannia cuneatissima, H. minuticarpa y $H$. arqueonervosa) con las cuales también está relacionada, pero de cuales difiere por presentar flores blancas además de otros caracteres (Fig. 1, Cuadro 1).

\section{CONTRIBUCIONES DE AUTORES}

GCC concibió y diseñó el estudio, participó en la revisión de los especímenes, diseñó la integración de la matriz de datos, realizó los análisis e interpretación de los mismos y escribió el manuscrito. ABB participó en la revisión de los ejemplares, en la captura de los datos, en el análisis de los mismos y en la escritura del manuscrito.

\section{FINANCIAMIENTO}

Este trabajo se desarrolló con el apoyo del Instituto de Ecología, A.C. (proyecto 20030-10134 GCC).

\section{AGRADECIMIENTOS}

Nuestro agradecimiento a E. Saavedra por la elaboración de la ilustración y a Ma. Elena Medina Abreo por sus atinados comentarios. A los curadores de los herbarios CHAPA, ENCB, MEXU y XAL por permitirnos examinar las colecciones, a los revisores anónimos que gracias a sus observaciones logramos esclarecer mejor esta contribución.

\section{LITERATURA CITADA}

Borhidi, A. 2006. Rubiáceas de México. Académiai Kiadó. Budapest, Hungría. 512 pp.

Borhidi, A. 2012. Rubiáceas de México. Académiai Kiadó. Budapest, Hungría. 608 pp.

Burger, W. C. 1999. Two new species of Hoffmannia (Rubiaceae) from Panama, with remarks on the circumscription of Mesoamerican species of the genus. Novon 9(1): 13-17. DOI: http://dx.doi.org/10.2307/3392108

Burger, W. C. y C. M. Taylor. 2012. Hoffmannia Sw. Rubiaceae a Verbenaceae. Flora Mesoamericana 4(2): 1-533.

Dwyer, J. D. 1969. The genus Hoffmannia (Rubiaceae) in Panama. Annals of the Missouri Botanical Garden 56(2): 269286. DOI: http://dx.doi.org/10.2307/2394842

Dwyer, J. D. 1980. Rubiaceae. Flora of Panama. Annals of the Missouri Botanical Garden 67: 1-522.

García, E. 1988. Modificaciones al sistema de clasificación climática de Köppen. Ed. Offset Larios. México, D.F., México. 252 pp.

González, A. L. y L. Poveda. 2004. Hoffmannia stephaniae (Rubiaceae), una nueva especie de Costa Rica. Lankesteriana 4(3): 183-185. DOI: http://dx.doi.org/10.15517/ lank.v4i3.21255

Kiehn, M. 1995. Chromosome survey of the Rubiaceae. Annals of the Missouri Botanical Garden 82(3): 398-408. DOI: http://dx.doi.org/10.2307/2399890

Kovach, W. 1999. MVSP-a Multivariate Statistical Package for Windows, version 3.1. Kovach Computing Services. Pentraeth, UK.

Miranda, F. y E. Hernández X. 1963. Los tipos de vegetación de México y su clasificación. Boletín de la Sociedad Botánica de México 28: 29-179. 
Sneath, R. y R. Sokal. 1973. Numerical taxonomy, the principles and practice of numerical classification. W. H. Freeman and Co. San Francisco, USA. 573 pp.

Standley, P. C. 1934. Rubiaceae. North America Flora 32(3-4): 159-300.

Standley, P. C. y L. O. Williams. 1975. Rubiaceae. Flora of Guatemala. Fieldiana, Botany 24(XI:1/3): 1-274. DOI: http://dx.doi.org/10.5962/bhl.title.2421
Taylor, C. M. y R. E. Gereau. 2011. Rubiacearum Americanarum magna hama pars XXVI: New species of Hoffmannia (Hamelieae) and more comments on the genus. Novon 21(1): 94-117. DOI: http://10.3417/2009118

Williams, L. 1973. Hoffmannia from Mexico and Central America. Fieldiana, Botany 36(6): 51-60. DOI: http://dx. doi.org/10.5962/bhl.title. 2555 